\title{
Ambiguities in one-dimensional phase retrieval from magnitudes of a linear canonical transform
}

\author{
Robert Beinert \\ Institut für Numerische und Angewandte Mathematik \\ Georg-August-Universität Göttingen
}

\begin{abstract}
Phase retrieval problems occur in a wide range of applications in physics and engineering. Usually, these problems consist in the recovery of an unknown signal from the magnitudes of its Fourier transform. In some applications, however, the given intensity arises from a different transformation such as the Fresnel or fractional Fourier transform. More generally, we here consider the phase retrieval of an unknown signal from the magnitudes of an arbitrary linear canonical transform. Using the close relation between the Fourier and the linear canonical transform, we investigate the arising ambiguities of these phase retrieval problems and transfer the well-known characterizations of the solution sets from the classical Fourier phase retrieval problem to the new setting.
\end{abstract}

Key words: Phase retrieval; One-dimensional signals; Compact support; Linear canonical transform

AMS Subject classifications: 42A05, 94A08, 94A12

\section{Introduction}

In many applications in physics and engineering such as crystallography [Mil90, Hau91, astronomy [BS79, DF87], and laser optics [SST04, SSD ${ }^{+}$06], one is faced with the socalled phase retrieval problem. The one-dimensional varient of this problem consists in the recovery of an unknown signal $f: \mathbb{R} \rightarrow \mathbb{C}$ in $L^{2}(\mathbb{R})$ with compact support from its Fourier intensity

$$
|\mathcal{F}[f](\omega)|:=\left|\int_{-\infty}^{\infty} f(t) \mathrm{e}^{-\mathrm{i} \omega t} \mathrm{~d} t\right| .
$$

Physically, one can interpret these magnitudes as intensity measurements of a wave in the far field. If one measures the intensity in the near field, one has to replace the Fourier transform by the Fresnel or the fractional Fourier transform Goo96]. In order to investigate the occurring ambiguities of the corresponding phase retrieval problems, we exploit that all three transformations - Fourier, Fresnel, and fractional Fourier transform - are special cases of the linear canonical transform [Wol79, Chap. 9]. 


\section{The linear canonical transform}

For the real parameters $a, b, c$, and $d$ with $a d-b c=1$, the linear canonical transform is defined by

$$
\mathcal{C}_{(a, b, c, d)}[f](\omega):=\int_{-\infty}^{\infty} f(t) K_{(a, b, c, d)}(\omega, t) \mathrm{d} t
$$

with the kernel

$$
K_{(a, b, c, d)}(\omega, t):= \begin{cases}\frac{1}{\sqrt{2 \pi b}} \mathrm{e}^{-\mathrm{i} \frac{\pi}{4}} \mathrm{e}^{\frac{\mathrm{i}}{2}\left(\frac{a}{b} t^{2}-\frac{2}{b} \omega t+\frac{d}{b} \omega^{2}\right)} & b \neq 0, \\ \frac{1}{\sqrt{a}} \mathrm{e}^{\mathrm{i} \frac{c}{2 a} \omega^{2}} \delta\left(t-\frac{\omega}{a}\right) & \text { else }\end{cases}
$$

where $\delta$ denotes the Dirac delta-function, see [Wol79, Chap. 9].

Obviously, the linear canonical transform $\mathcal{C}_{(0,1,-1,0)}$ is identical to the Fourier transform $\mathcal{F}$ up to the multiplicative constant

$$
\theta:=\theta_{(a, b, c, d)}:=\frac{1}{\sqrt{2 \pi b}} \mathrm{e}^{-\mathrm{i} \frac{\pi}{4}} .
$$

Moreover, the linear canonical transform covers a complete family of well-known integral transformations. For instance, $\mathcal{C}_{(1,1 / 2 \alpha, 0,1)}$ and $\mathcal{C}_{(\cos \alpha, \sin \alpha,-\sin \alpha, \cos \alpha)}$ with $\alpha \in \mathbb{R}$ coincide with the Fresnel transform Gor81] and with the fractional Fourier transform [PD01, respectively.

If $b \neq 0$, the linear canonical transform can be written in the form

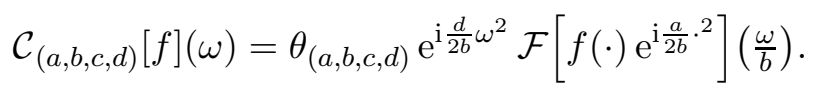

Using this relation to the Fourier transform, one can easily show that the inverse linear canonical transform is given by

$$
\mathcal{C}_{(a, b, c, d)}^{-1}[\widetilde{f}](t)=\int_{-\infty}^{\infty} \widetilde{f}(\omega) \overline{K_{(a, b, c, d)}(\omega, t)} \mathrm{d} \omega,
$$

which coincides up to a unimodular constant with $\mathcal{C}_{(d,-b,-c, a)}$.

\section{Phase retrieval from magnitudes of the linear canonical transform}

We now consider the corresponding phase retrieval problem. In other words, we wish to recover a signal $f \in L^{2}(\mathbb{R})$ with compact support from $\left|\mathcal{C}_{(a, b, c, d)}[f]\right|$. Since $\mathcal{C}_{(a, 0, c, d)}[f]$ is merely a scaled and modulated version of $f$, and since the recovery of a complex-valued function is not possible from its modulus in general, we assume that $b \neq 0$. Similarly to the Fourier setting, this phase retrieval problem cannot be solved uniquely. 
Proposition 3.1. Let $f \in L^{2}(\mathbb{R})$ be a signal with compact support. Then

(i) the rotated signal $\mathrm{e}^{\mathrm{i} \alpha} f$ with $\alpha \in \mathbb{R}$

(ii) the shifted signal $\mathrm{e}^{-\mathrm{i} a t_{0} / b} f\left(\cdot-t_{0}\right)$ with $t_{0} \in \mathbb{R}$

(iii) the reflected signal $\mathrm{e}^{-\mathrm{i} a \cdot 2 / b} \overline{f(-\cdot)}$

have the same linear canonical intensity $\left|\mathcal{C}_{(a, b, c, d)}[f]\right|$.

Proof. The assertion can be established by applying (3) and using the properties of the Fourier transform.

(i) $\mathcal{C}_{(a, b, c, d)}\left[\mathrm{e}^{\mathrm{i} \alpha} f\right](\omega)=\mathrm{e}^{\mathrm{i} \alpha} \mathcal{C}_{(a, b, c, d)}[f](\omega)$

(ii) $\mathcal{C}_{(a, b, c, d)}\left[\mathrm{e}^{-\mathrm{i} a t_{0} / b} f\left(\cdot-t_{0}\right)\right](\omega)=\theta \mathrm{e}^{-\mathrm{i} a t_{0}^{2} / 2 b} \mathrm{e}^{\mathrm{i} d \omega^{2} / 2 b} \mathcal{F}\left[f\left(\cdot-t_{0}\right) \mathrm{e}^{\mathrm{i} a\left(\cdot-t_{0}\right)^{2} / 2 b}\right](\omega / b)$

$$
=\mathrm{e}^{-\mathrm{i} a t_{0}^{2} / 2 b} \mathrm{e}^{-\mathrm{i} \omega t_{0} / b} \mathcal{C}_{(a, b, c, d)}[f](\omega)
$$

(iii) $\mathcal{C}_{(a, b, c, d)}\left[\mathrm{e}^{-\mathrm{i} a \cdot 2 / b} \overline{f(-\cdot)}\right](\omega)=\theta \mathrm{e}^{\mathrm{i} d \omega^{2} / 2 b} \mathcal{F}\left[\overline{f(-\cdot) \mathrm{e}^{\mathrm{i} a(-\cdot)^{2} / 2 b}}\right](\omega / b)$

$$
=\mathrm{e}^{2 \mathrm{i} \arg \theta} \mathrm{e}^{\mathrm{i} d \omega^{2} / b} \overline{\mathcal{C}_{(a, b, c, d)}[f](\omega)}
$$

Considering the absolute value of each equation finishes the proof.

Without further information about the unknown signal, these three ambiguities cannot be avoided. Considering that these signals are, however, closely related to the original signal $f$, we call them trivial ambiguities. Besides these ambiguities, the phase retrieval problem usually possesses a series of further non-trivial ambiguities. Using (3), the complete solution set can be characterized similarly to the Fourier case in Wal63, Hof64, Bei16. For this, we denote the Laplace transform and the autocorrelation function of a signal $f \in L^{2}(\mathbb{R})$ by

$$
\mathcal{L}[f](\zeta):=\int_{-\infty}^{\infty} f(t) \mathrm{e}^{-\zeta t} \mathrm{~d} t \quad \text { and } \quad A[f](\zeta):=\int_{-\infty}^{\infty} \int_{-\infty}^{\infty} \overline{f(s)} f(s+t) \mathrm{e}^{-\zeta t} \mathrm{~d} s \mathrm{~d} t
$$

Theorem 3.2. Let $f \in L^{2}(\mathbb{R})$ be a signal with compact support. Then each signal $g \in$ $L^{2}(\mathbb{R})$ with compact support and $\left|\mathcal{C}_{(a, b, c, d)}[g]\right|=\left|\mathcal{C}_{(a, b, c, d)}[f]\right|$ is of the form

$$
\mathcal{L}\left[\theta \mathrm{e}^{\frac{\mathrm{i} a}{2 b} \cdot 2} g\right](\zeta)=C \zeta^{m} \mathrm{e}^{\zeta \gamma} \prod_{j=1}^{\infty}\left(1-\frac{\zeta}{\eta_{j}}\right) \mathrm{e}^{\frac{\zeta}{\eta_{j}}},
$$

where the absolute value $|C|$ and the imaginary part $\Im \gamma$ of the complex constants $C$ and $\gamma$ coincide for all signals $g$, and where $\eta_{j}$ is chosen from the zero pair $\left(\xi_{j}, \bar{\xi}_{j}\right)$ of $A\left[\theta \mathrm{e}^{\mathrm{i} a \cdot 2 / 2 b} f\right]$.

Proof. Since $\left|\mathcal{C}_{(a, b, c, d)}[f](\omega)\right|=\left|\theta \mathcal{F}\left[f \mathrm{e}^{\mathrm{i} a .{ }^{2} / 2 b}\right](\omega / b)\right|$, we can identify the phase retrieval problem to recover $f$ from $\left|\mathcal{C}_{(a, b, c, d)}[f]\right|$ with the phase retrieval problem to recover 
$\theta f \mathrm{e}^{\mathrm{i} a \cdot{ }^{2} / 2 b}$ from $\left|\theta \mathcal{F}\left[f \mathrm{e}^{\mathrm{ia} \cdot{ }^{2} / 2 b}\right]\right|$. Hence, the solutions of both problems differ only by $\theta \mathrm{e}^{\mathrm{i} a \cdot 2} / 2 b$. Using [Bei16, Theorem 3.3] to characterize the solutions of the Fourier phase retrieval problem, we immediately obtain the assertion.

Remark 3.3. Since we have assumed that the unknown signal $f$ has a compact support, the autocorrelation function $A\left[\theta \mathrm{e}^{\mathrm{i} a .{ }^{2} / 2 b} f\right]$ in Theorem 3.2 is the analytic continuation of $\left|\mathcal{F}\left[\theta \mathrm{e}^{\mathrm{i} a \cdot{ }^{2} / 2 b} \mathrm{f}\right]\right|^{2}$ from the complex axis to the complex plane, i.e.

$$
A\left[\theta \mathrm{e}^{\mathrm{i} \frac{a}{2 b} \cdot{ }^{2}} f\right](\mathrm{i} \omega)=\left|\mathcal{F}\left[\theta \mathrm{e}^{\mathrm{i} \frac{a}{2 b} \cdot{ }^{2}} f\right](\omega)\right|^{2} \quad(\omega \in \mathbb{R}),
$$

see for instance [Bei16, Proposition 3.2]. Hence, the required autocorrelation function $A\left[\theta \mathrm{e}^{\mathrm{i} a .{ }^{2} / 2 b} f\right]$ is completely encoded in the given intensity $\left|\mathcal{C}_{(a, b, c, d)}[f]\right|$.

\section{Discretization of the linear canonical phase retrieval problem}

To determine a numerical solution, one has to discretize the problem formulation. For this purpose, we replace the continuous-time signal $f: \mathbb{R} \rightarrow \mathbb{C}$ by a discrete-time signal $x: \mathbb{Z} \rightarrow \mathbb{C}$. Analogously to the continuous-time setting, we assume that the signal $x \in \ell^{2}(\mathbb{Z})$ has a finite support, which means that only finitely many signal components $x[n]$ are non-zero. Discretizing the integral in (11), we define the linear canonical transform of the signal $x:=(x[n])_{n \in \mathbb{Z}}$ by

$$
\mathcal{C}_{(a, b, c, d)}[x](\omega):=\sum_{n \in \mathbb{Z}} x[n] K_{(a, b, c, d)}(\omega, n),
$$

where $K_{(a, b, c, d)}$ is again the kernel in (2).

Analogous to (3), the linear canonical transform can be written as

$$
\mathcal{C}_{(a, b, c, d)}[x](\omega)=\theta_{(a, b, c, d)} \mathrm{e}^{\mathrm{i} \frac{d}{2 b} \omega^{2}} \mathcal{F}\left[x[\cdot] \mathrm{e}^{\mathrm{i} \frac{a}{2 b} \cdot{ }^{2}}\right]\left(\frac{\omega}{b}\right)
$$

whenever $b \neq 0$. Here $\mathcal{F}$ denotes the discrete-time variant of the Fourier transform given by

$$
\mathcal{F}[x](\omega):=\sum_{n \in \mathbb{Z}} x[n] \mathrm{e}^{-\mathrm{i} \omega n}
$$

Reversing (44), we notice that the discrete-time linear canonical transform can be inverted by

$$
\mathcal{C}_{(a, b, c, d)}^{-1}[\widetilde{x}][n]=\int_{-\pi|b|}^{\pi|b|} \widetilde{x}(\omega) \overline{K(\omega, n)} \mathrm{d} \omega .
$$

Based on our definitions, the discrete-time variant of the phase retrieval problem can be stated as follows: recover the unknown discrete-time signal $x \in \ell^{2}(\mathbb{Z})$ with finite support from $\left|\mathcal{C}_{(a, b, c, d)}[x]\right|$. For the same reason as before, we assume that $b \neq 0$. Adapting the 
proof of Proposition 3.1, we can simply transfer the three kinds of trivial ambiguities to the discrete-time setting.

Proposition 4.1. Let $x \in \ell^{2}(\mathbb{Z})$ be a signal with finite support. Then

(i) the rotated signal $\mathrm{e}^{\mathrm{i} \alpha} x$ with $\alpha \in \mathbb{R}$

(ii) the shifted signal $\mathrm{e}^{-\mathrm{i} a n_{0} / b} x\left[\cdot-n_{0}\right]$ with $n_{0} \in \mathbb{Z}$

(iii) the reflected signal $\mathrm{e}^{-\mathrm{i} a \cdot 2 / b} \overline{x[-\cdot]}$

have the same linear canonical intensity $\left|\mathcal{C}_{(a, b, c, d)}[x]\right|$.

In order characterize the non-trivial ambiguities, we will exploit the representations of the non-trivial solutions of the Fourier phase retrieval problem in [BS79, BP15]. Denoting the support length of the discrete-time signal $x \in \ell^{2}(\mathbb{Z})$ by $N$, we define the corresponding autocorrelation signal and autocorrelation polynomial by

$$
a[x][n]:=\sum_{k \in \mathbb{Z}} x[k] \overline{x[k+n]} \quad \text { and } \quad P_{A}[x](z):=z^{N-1} \sum_{n=-N+1}^{N-1} a[x][n] z^{n} .
$$

Since the autocorrelation signal $a[x]$ possesses the support $\{-N+1, \ldots, N-1\}$, the autocorrelation polynomial $P_{A}[x]$ is always a well-defined polynomial of degree $2 N-2$.

Theorem 4.2. Let $x \in \ell^{2}(\mathbb{Z})$ be a signal with finite support. Then each signal $y \in \ell^{2}(\mathbb{Z})$ with finite support and $\left|\mathcal{C}_{(a, b, c, d)}[y]\right|=\left|\mathcal{C}_{(a, b, c, d)}[x]\right|$ is of the form

$$
\mathcal{F}\left[\theta \mathrm{e}^{\frac{\mathrm{i} a}{2 b} \cdot{ }^{2}} y\right](\omega)=\mathrm{e}^{\mathrm{i}\left(\alpha+\omega n_{0}\right)} \sqrt{\left|a\left[\theta \mathrm{e}^{\mathrm{i} \frac{a}{2 b} \cdot 2} x\right][N-1]\right| \prod_{j=1}^{N-1}\left|\beta_{j}\right|-1} \cdot \prod_{j=1}^{N-1}\left(\mathrm{e}^{-\mathrm{i} \omega}-\beta_{j}\right)
$$

where $\alpha \in \mathbb{R}, n_{0} \in \mathbb{Z}$, and $\beta_{j}$ is chosen from the zero pair $\left(\gamma_{j}, \bar{\gamma}_{j}^{-1}\right)$ of $P_{A}\left[\theta \mathrm{e}^{\mathrm{i} a \cdot 2 / 2 b} x\right]$ for $j=1, \ldots, N-1$.

Proof. Similarly to the equivalent continuous-time statement, the relationship (4) implies that $\left|\mathcal{C}_{(a, b, c, d)}[x](\omega)\right|=\left|\theta \mathcal{F}\left[x \mathrm{e}^{\mathrm{i} a .{ }^{2} / 2 b}\right](\omega / b)\right|$. We can thus reduce the considered phase retrieval problem to the recovery of $\theta x \mathrm{e}^{\mathrm{i} a \cdot{ }^{2} / 2 b}$ from $\left|\theta \mathcal{F}\left[x \mathrm{e}^{\mathrm{i} a \cdot 2 / 2 b}\right]\right|$. Now, the assertion immediately follows from the characterization of the solution set of the Fourier phase retrieval problem in [BP15, Theorem 2.4].

Remark 4.3. Considering the well-known relation

$$
\mathcal{F}\left[a\left[\theta x \mathrm{e}^{\mathrm{i} \frac{a}{2 b} \cdot 2}\right]\right]=\left|\mathcal{F}\left[\theta x \mathrm{e}^{\mathrm{i} \frac{a}{2 b} \cdot{ }^{2}}\right]\right|^{2}=\left|C_{(a, b, c, d)}[x]\right|^{2}
$$

between the autocorrelation signal and the squared Fourier intensity of a discrete-time signal with finite support, see for instance [BP15, p. 1173], the autocorrelation polynomial $P_{A}\left[\theta \mathrm{e}^{\mathrm{i} a \cdot{ }^{2} / 2 b} x\right]$ in Theorem 4.2 is completely determined by $\left|\mathcal{C}_{(a, b, c, d)}[x]\right|$. 
Moreover, equation (5) shows that $\left|\mathcal{C}_{(a, b, c, d)}[x]\right|^{2}$ as Fourier transform of the autocorrelation signal $a\left[\theta \mathrm{e}^{\mathrm{i} a \cdot{ }^{2} / 2 b} x\right]$ is a non-negative real-valued trigonometric polynomial of degree $N-1$. Hence, the intensity $\left|\mathcal{C}_{(a, b, c, d)}[x]\right|$ is already uniquely defined by $2 N-1$ samples at appropriate points in $[-\pi, \pi)$.

\section{Conclusion}

Using the close relation between the linear canonical transform and the Fourier transform, we have characterized the complete solution set of the phase retrieval problem to recover a continuous-time or discrete-time signal from the intensity of its linear canonical transform. With the same approach, one can transfer most of the uniqueness results for the classical phase retrieval problem, see for instance [BFGR76, KST95, RDN13, BP15, Bei16, BP16] and references therein, to the new setting.

\section{Acknowledgements}

I gratefully acknowledge the funding of this work by the DFG in the framework of the SFB 755 'Nanoscale photonic imaging' and of the GRK 2088 'Discovering structure in complex data: Statistics meets Optimization and Inverse Problems.'

\section{References}

[Bei16] BEINERT, Robert: One-dimensional phase retrieval with additional interference measurements. April 2016. - Preprint, arXiv:1604.04489v1

[BFGR76] Burge, R. E. ; Fiddy, M. A. ; Greenaway, A. H. ; Ross, G.: The phase problem. In: Proceedings of the Royal Society of London. Series A. Mathematical Physical \&5 Engineering Sciences 350 (1976), pp. 191-212

[BP15] Beinert, Robert ; PlonkA, Gerlind: Ambiguities in one-dimensional discrete phase retrieval from Fourier magnitudes. In: Journal of Fourier Analysis and Applications 21 (2015), December, No. 6, pp. 1169-1198

[BP16] Beinert, Robert ; PlonkA, Gerlind: Enforcing uniqueness in one-dimensional phase retrieval by additional signal information in time domain. March 2016. - Preprint, arXiv:1604.04493v1

[BS79] Bruck, Yu. M. ; Sodin, L. G.: On the ambiguity of the image reconstruction problem. In: Optics communications 30 (1979), September, No. 3, pp. 304-308

[DF87] DAinty, J. C. ; Fienup, J. R.: Phase retrieval and image reconstruction for astronomy. In: Stark, Henry (Ed.): Image Recovery : Theory and Application. Orlando (Florida) : Academic Press, 1987, Chapter 7, pp. 231-275

[Goo96] Goodman, Joseph W.: Introduction to Fourier Optics. 2nd Edition. New York : McGrawHill, 1996 (McGraw-Hill Series in Electrical and Computer Engineering : Electromagnetics)

[Gor81] Gori, F.: Fresnel transform and sampling theorem. In: Optics Communications 39 (1981), November, No. 5, pp. 293-297

[Hau91] Hauptman, Herbert A.: The phase problem of x-ray crystallography. In: Reports on Progress in Physics 54 (1991), November, No. 11, pp. 1427-1454

[Hof64] Hofstetter, Edward M.: Construction of time-limited functions with specified autocorrelation functions. In: IEEE Transaction on Information Theory 10 (1964), April, No. 2, pp. $119-126$ 
[KST95] Klibanov, Michael V. ; Sacks, Paul E. ; Tikhonravov, Alexander V.: The phase retrieval problem. In: Inverse Problems 11 (1995), No. 1, pp. 1-28

[Mil90] Millane, R. P.: Phase retrieval in crystallography and optics. In: Journal of the Optical Society of America A 7 (1990), March, No. 3, pp. 394-411

[PD01] PEI, Soo-Chang ; DING, Jian-Jiun: Relations between fractional operations and timefrequency distributions, and their applications. In: IEEE Transactions on Signal Processing 49 (2001), August, No. 8, pp. 1638-1655

[RDN13] Raz, Oren ; Dudovich, Nirit ; Nadler, Boaz: Vectorial phase retrieval of 1-D signals. In: IEEE Transactions on Signal Processing 61 (2013), April, No. 7, pp. 1632-1643

[SSD ${ }^{+}$06] Seifert, Birger ; Stolz, Heinrich ; Donatelli, Marco ; Langemann, Dirk ; Tasche, Manfred: Multilevel Gauss-Newton methods for phase retrieval problems. In: Journal of Physics. A. Mathematical and General 39 (2006), No. 16, pp. 4191-4206

[SST04] Seifert, Birger ; Stolz, Heinrich ; TAsche, Manfred: Nontrivial ambiguities for blind frequency-resolved optical gating and the problem of uniqueness. In: Journal of the Optical Society of America B 21 (2004), May, No. 5, pp. 1089-1097

[Wal63] Walther, Adriaan: The question of phase retrieval in optics. In: Optica Acta: International Journal of Optics 10 (1963), No. 1, pp. 41-49

[Wol79] Wolf, Kurt B.: Integral Transforms in Science and Engineering. New York : Plenum Press, 1979 